\title{
Effects of estrogen benzoate on the microanatomy of adrenal gland in female Albino rats
}

\author{
Mohd Saleem Itoo $^{1 *}$, Shaheen Shahdad ${ }^{2}$, Omer Bashir Itoo ${ }^{3}$, Fahmida Akhter ${ }^{3}$, Naseer Ahmad Shah ${ }^{4}$, Bashir \\ Ahmad Shah ${ }^{1}$, Gh.Mohd Bhat ${ }^{5}$, Arshid Hussain Zagoo ${ }^{4}$, Suhail Ahmad Gilkar ${ }^{4}$ \\ $I^{*}$ Assistant Professor Department of Anatomy Government Medical College Srinagar J\&K, India \\ ${ }^{2}$ Professor \& Head Department of Anatomy Government Medical College Srinagar J\&K, India \\ ${ }^{3}$ Assistant Surgeon / Demonstrator Government Medical College Srinagar J\&K, India \\ ${ }^{4}$ Lecturer Department of Anatomy/Physiology Government Medical College Srinagar $J \& K$, India \\ ${ }^{5}$ Associate Professor Department of Anatomy Government Medical College Srinagar J\&K, India \\ *Corresponding author E-mail: dr.saleem68@gmail.com
}

\begin{abstract}
The present study was conducted in the department of Anatomy Government Medical College Srinagar to observe the effect of graded doses of estrogen benzoate on the microanatomy of adrenal gland in female Albino rats by giving variable doses of estrogen to these animals for variable periods. Sixty female albino rats weighing on an average $100 \mathrm{gms}$ were used for this study. The animals taken from animal house of Government Medical College Srinagar were divided randomly into three groups. Group A served as control, Group B and C received daily low and high doses of estrogen benzoate diluted in coconut oil respectively. From each group animals were sacrificed at intervals of one, three, six and twelve weeks.5-6 micrometer thick histological sections of adrenal gland were cut, fixed on glass slides and stained with Haematoxyline \& Eosin. Microscopic changes in the adrenal gland were recorded. It was observed that estrogen benzoate produces dose and duration dependent histopathological changes in the adrenal gland of female albino rats in the form of thickening of the glandular capsule, dilatation and congestion of sinusoids both in the cortex and medulla of the gland.
\end{abstract}

Keywords: Adrenal cortex, estrogen benzoate, female Albino rats, haemorrhages, sinusoids.

\section{Introduction}

Estrogens are substances which produce estrous in spayed animals. They are synthesized from cholesterol. In nonpregnant female major quantities of estrogens are secreted by ovaries and minor quantities are produced by adrenal cortex, tremendous quantities of it are produced by placenta during pregnancy and majority of it is excreted in urine after undergoing conjugation in liver. Human urine of pregnancy is thus an abundant source of natural estrogen. The pregnant mare excretes about 100 micrograms of estrogen daily. Among living creatures stallion who despite manifestations of virility excretes into its environment more estrogen than any other animal. In male estrogens are produced by testes. Estrogens are widely prescribed steroids. Some food stuffs like soy, grapes and its extract like wine and some cosmetics of our daily use like skin creams and hair dyes contain variable amounts of estrogen. Alfalfa, animal flesh, Anise seed, Apples, Baker's yeast Bareley, Beets, Carrots, Cherries, Chickpeas, Clover, Cowpeas (blackeyed peas). Cucumbers, Dairy foods, Dates, Eggs, Eggplant. Fennel, Flaxseeds, Garlic, Hops, Licorice, Oats, Olive oil ,Olives, Papaya , Parsley , Peas , Peppers Plums Pomegranates, Potatoes, Pumpkin Red beans , Red clover , Rhubarb, Rice Sage. Sesame, Soybean, Sprouts, Soybeans, Split peas, Sunflower seeds, Tomatoes, Wheat, Yams, Soybeans, and soy derivatives have large amounts of natural estrogens. Flaxseed, pulses, citrus fruits, wheat and licorice also have fairly large amounts of natural estrogens. A normal woman secretes about 2800 micrograms of estrogen monthly. Out of this about 700 micrograms are excreted in urine. A woman who is taking an ordinary contraceptive pill containing about 50 micrograms of ethinyl estradiol absorbs about 30 micrograms per day i.e, 600ugms/month. This is for less than the naturally secreted estrogen. Estrogens are absorbed by oral, parenteral and transdermal routes. Liver plays a key role in its metabolism. It conjugates estrogen to form glucuronides and sulfates which are mainly excreted in urine. Estrogen exerts its effects on many tissues of body by acting on estrogenic receptors [1]. Thus present research was under taken to study the effect of graded doses of estrogen benzoate on the microanatomy of adrenal gland which shows increase in wet weight and vascularity after estrogen administration. 


\section{Materials and methods}

Sixty female albino rats weighing on an average 100 grams were taken from animal house of Government Medical College Srinagar for the present study. These animals were divided into three groups.

Group A---12 rats (Control Group) were given normal diet and no drug.

Group B---24 rats were injected $0.05 \mathrm{mg}$ of estrogen benzoate daily.

Group C---24 rats were injected $0.2 \mathrm{mg}$ of estrogen benzoate daily.

All rats were kept under uniform husbandry conditions in 60 iron cages separately. They were fed with normal diet consisting of Gram, vegetables and tap water. One milliliter of injection estrogen benzoate ( $5 \mathrm{mg}$ ) was diluted with $4 \mathrm{ml}$ of coconut oil i.e., each $\mathrm{ml}$ of diluted oil contains $01 \mathrm{mg}$ of estrogen benzoate. One ml of diluted drug was taken in subcutaneous (insulin) syringe containing 40 graduations i.e. 01 graduation $=0.025 \mathrm{mgs}$. Group B animals were injected 2 graduations of drug $(0.05 \mathrm{mg})$ daily while as group $\mathrm{C}$ animals received eight graduations $(0.2 \mathrm{mg})$ daily. This process of drug administration was continued regularly for 12 weeks. The animals were sacrificed in four sittings i.e. after intervals of one, three, six and twelve weeks. In each sitting three rats from group A, six rats each from group B and C were taken out of their cages after anesthetizing with chloroform. The limbs of each rat were fixed on board with pins. A midline incision was given on anterior abdominal wall. Adrenal glands were identified, cleaned, dissected out and put in dishes containing formal saline. These tissues were processed manually for block making using standard histological techniques. Sections measuring 5-6 micrometers were cut and fixed on glass slides. The sections were stained with Haematoxyline \& Eosin. The microscopic observations were recorded group wise using a Compound light microscope. Appropriate photographs were taken using photographic Microscope and labeled.

\section{Observations}

On gross examination adrenal gland was pea nut shaped, yellow in colour and hard in consistency with smooth surface in all groups of animals. Following microscopic observations were recorded.

Control group: In this group cut section of adrenal gland showed the usual microscopic structure consisting of outer cortex and inner medulla. Cortex was divided into three zones from without inwards, Zona Glomerulosa (Sub capsular Zone), Zona Fasciculata (middle Zone) and Zona Reticularis (inner most zones).The gland was enclosed in a welldefined capsule. Medulla of the gland is composed of cords of cells surrounding sinusoids as shown in fig.1. No changes in the microscopic anatomy of adrenal gland were observed at any stage of this study in the control group (A).

Group B and Group C: The changes in the microscopic structure began to appear after second week of drug administration in the form of increase in thickness of the capsule and vascularity of the gland. Sinusoids were dilated and congested both in cortex and medulla. These changes were marked after 12 weeks of drug treatment as shown in fig. 2 .

\section{Discussion}

Harrison \& Hoey (1960) [2] found the suprarenal gland in humans is very vascular structure and is supplied by three groups of arteries namely the superior, middle and inferior supra renal arteries. Most suprarenal branches ramify over the capsule before entering the gland to form sub capsular plexus.

Effect of estrogen on the histopathology of adrenal gland has not received much consideration. Richards R.K. Kenter (1941) [3] found that chronic adminstration of diethyl stilbestrol caused growth retardation and occasional adrenal haemorrhages in female albino rats. Morikawa Y, Hashimoto,Eguchi Y. (1976)[4] studied the effect of estradiol benzoate on the adrenal glands of rats, by giving this drug diluted with Sesame oil 2 hrs after birth to these animals and found that it fails to shrink neonatal rat adrenal gland when given soon after birth. Saruhan BG,Ozdemir N (2002)[5] observed the effect of estrogen on adrenal gland histology to ascertain whether or not estrogen regulates body and adrenal gland wet weight gain in the ovariectomized rat model. Two experiments investigated the effects of ovariectomy and estrogen replacement on the body and adrenal gland weight by using 36 female Wistar Albino rats divided into three groups. Group 1 (control group) received no ovariectomy; all animals in the other groups were bilaterally ovariectomized and kept for 60 days. They designated group 2 and 3 as sham-operated group and bilaterally ovariectomized then in addition, group 3 received estradiol. Then $5 \mathrm{~mm}$ thick paraffin sections, were obtained by routine histological methods, and stained with hematoxylin-eosin and later evaluated under a light microscope. They observed that the body weight was higher in the ovariectomized rats compared to the control groups. Ovariectomy did not result in significant changes in the wet weight of the adrenal gland. Furthermore, body weight increased after estrogen therapy, and the wet weight of the adrenal gland increased in the estrogen group. In the sham-operated and 
estrogen group, they observed a prominent capsule, expanded zona glomerulosa cells, regular parallel columns in zona fasciculata and there was an increased vascularization in the medulla, after the estrogen treatment.

In our present study we found that in estrogen benzoate treated albino rats, capsule of the gland was thickened. Sinusoids were dilated and congested both in cortex and medulla. Over all vascularity of the gland was increased. All these changes were marked in group $\mathrm{C}$ animals at various stages of research as compared to group B animals. No such microscopic changes were observed in animals of control group. Thus the observations made in our study are similar to those made by Morikawa Y,Hashimoto, Eguchi Y.(1976)[4], who reported that administration of estrogen benzoate given two hours after birth to neonatal mice fails to shrink the size of adrenal gland which in other words means that it causes increase in size of adrenal gland. Observation made by Saruhan BG,Ozdemir N. (2002)[5] are similar to our observations that administration of estrogen to rats causes thickening of capsule, increase in vascularity of both cortex and medulla of rat adrenal gland. However the observations made in our study are discordant with those made by Richards R.K Kenter (1941) [3] who found that chronic adminstration of diethyl stilbestrol caused growth retardation and occasional adrenal haemorrhages in female albino rats. Since we found increased vascularity (congestion) confined to the sinusoids of cortex and medulla, we label it as sinusoidal congestion rather than adrenal heamorrages as reported by Richards R.K Kenter (1941) [3].

\section{Conclusion}

Estrogen benzoate injection can cause a significant increase in the activity of the adrenal cortex and medulla. In our study we found that in estrogen treated animals capsule of the gland was thickened, Sinusoids were dilated and congested both in the cortex and medulla. Over all vascularity of the gland was increased. All these changes were marked in group $\mathrm{C}$ at various stages of research as compared to group B after 3rd week of estrogen adminstration. No such microscopic changes were observed in control group. We conclude that Estrogen benzoate produces definite histopathological changes in rat adrenal glands which are dose and duration dependent.

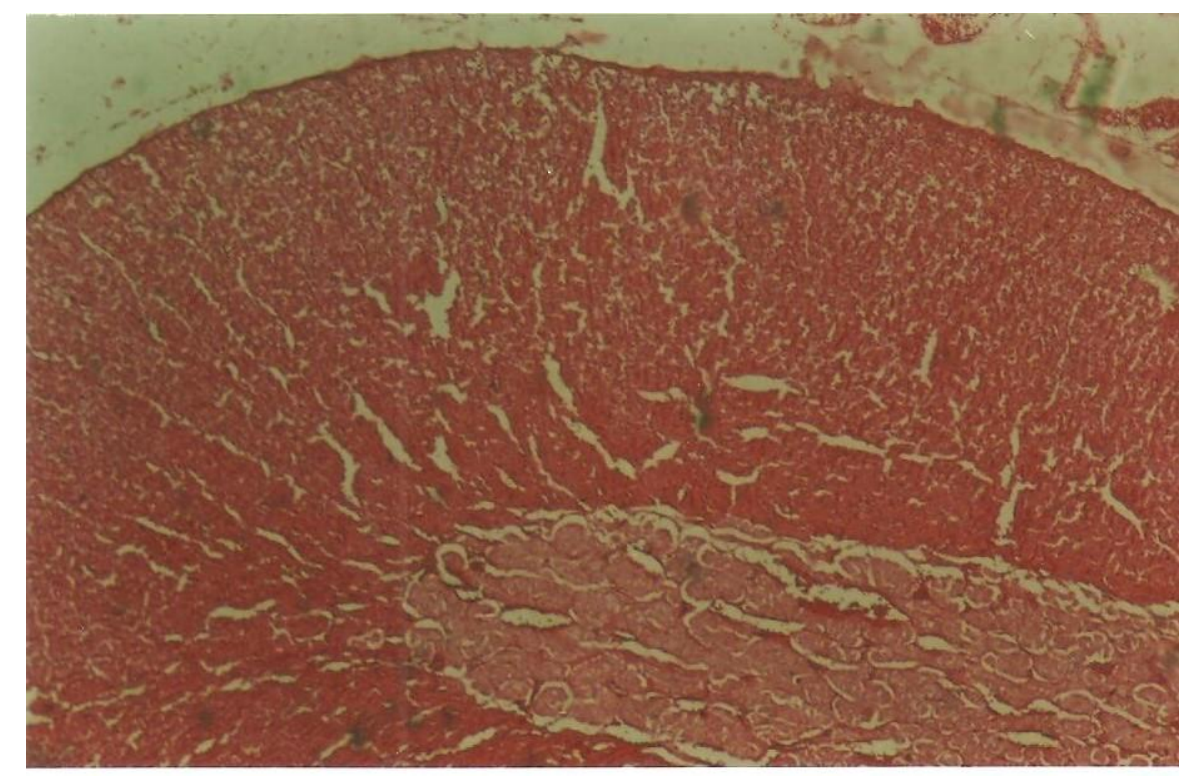

Fig.1: A microphotograph of adrenal gland from Control group ( A ) of female Albino Rats, showing capsule and normal sinusoids both in the cortex and medulla. 


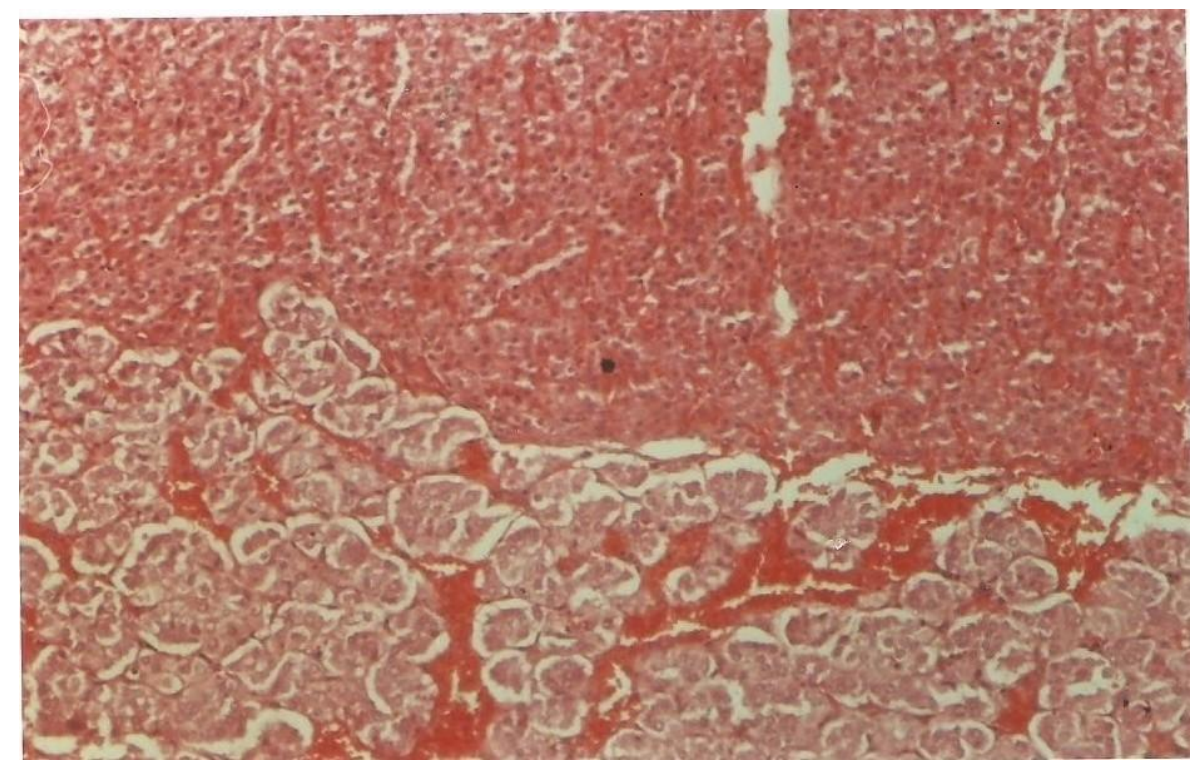

Fig.2: A microphotograph of adrenal gland from group (C) of female Albino rats after 12 weeks of Estrogen benzoate administration showing dilated and congested sinusoids both in the cortex and medulla. Stain: H\&E

Magnificati:on 100X

\section{References}

[1] Jensen E, V, Mechanism of action of female sex hormone, Annu Rev Biochem 1972, 41; 203-230

[2] Harrison \& Hoey, Endocrine System in Gray's Anatomy, The Anatomical Basis of Medicine And Surgery, $38^{\text {th }}$ Edition Edinburg Elsevier Churchill Living Stone. 2000; 1905.

[3] Richards R.K.Kenter, studies on the toxic effects of stilboestrol with special reference to growth retardation, Amn J.Physol 1941, $133,423$.

[4] Arishima K ,Morikawa Y,Hashimoto,Eguchi Y.(1976),Failure of estradiol benzoate given after birth to prevent the adrenal glands from shrinking in new born rats,Endocrinol,jpn.1976 Oct :23(5):429-30.

[5] Saruhan BG, Ozdemir N, Effect of ovarectomy and estrogen treatment on the adrenal gland and body weight in rats. Saudi medical j2006, Jan; 27(1):131. 